\title{
Laparoscopic Repair of Bilateral Inguinal Hernia Using Large Butterfly Mesh Without Fixation Versus Double Meshes with Stapler Fixation through TAPP Approach \\ Mahmoud Elsayed Nagaty \\ General Surgery, Faculty of Medicine, Al-Azhar university, Egypt
}

\begin{abstract}
Background: Inguinal hernia repair is one of the most frequent elective operations performed in general surgical practice. Approximately $20 \%$ to $30 \%$ of patients with inguinal hernia present with bilateral hernias at diagnosis.

Objectives: The aim of this study was to demonstrate the outcome between using a single large butterfly mesh without fixation to Strengthen the posterior wall versus the use of two separate meshes with stapler fixation during laparoscopic repair of bilateral inguinal hernia with TAPP technique.

Patients and Methods: A prospective randomized comparative study that was conducted in General Surgery Department, Al-Hussein University Hospital, Faculty of Medicine, Al-Azhar University from January 2017 to December 2018. A total of 40 patients with bilateral non-complicated inguinal hernias were included in this study. The patients were subsequently divided into two comparable groups. Group A: Twenty patients were scheduled for TAPP repair with double meshes and tacker fixation. Group B: Twenty patients were scheduled for TAPP repair with a single large mesh without fixation.

Results: Average operating time was 102 minutes in group A, and 72 minutes in group B. Postoperative pain was less in group B. One patient had transient hematuria in group B. There was one recurrence in group A on the left side after 8 months and no recurrences were demonstrated in group B.

Conclusion: laparoscopic TAPP using a single large butterfly mesh is safe, time saving and cost-effective approach for non-complicated bilateral inguinal hernias, especially in patients with direct hernia, large defect and low BMI.
\end{abstract}

Keywords: inguinal hernia, butterfly mesh, laparoscopic hernia repair.

\section{INTRODUCTION}

Inguinal hernia repair is one of the most frequent elective operations performed in general surgical practice ${ }^{(\mathbf{1})}$. Approximately $20 \%$ to $30 \%$ of patients with inguinal hernia present with bilateral hernias at diagnosis ${ }^{(2,3)}$. Although the indications of laparoscopic treatment of inguinal hernia remain controversial, most surgeons agree that patients with bilateral or recurrent inguinal hernia are those who benefit mostly from the laparoscopic approach, which is considered the gold standard for treating such cases ${ }^{(4-6)}$. The benefits of laparoscopic inguinal hernia repair include a decrease in postoperative pain, shorter hospital stay, and a small recovery Period ${ }^{(7)}$.

Laparoscopy can diagnose occult hernia in the contra-lateral side, also able to diagnose and treat recurrent and contra-lateral hernia at the same time (8). The recurrences after laparoscopic surgical repair has many causes such as incomplete and improper dissection, missed or undiagnosed hernia, using mesh with small size, mesh migration or bad poisoning of the mesh when the mesh is not fixed ${ }^{(9)}$.

Recently, the need for and best way of fixing the mesh have been questioned. Mesh fixation with staples or tacks is traditionally used and may reduce the risk of mesh displacement, and consequently, decrease hernia recurrence (10). However, fixation is associated with higher costs and may increase the rate of acute and especially chronic pain ${ }^{(11)}$.

\section{PATIENTS AND METHODS}

Forty patients with bilateral inguinal hernias (32 males and 8 females) were included in our study. The patients were subsequently randomly divided into two comparable groups according to surgeon and patient preference after thorough explanation of either techniques in relation to the type of hernia, body mass index and economic concern. Group A: Twenty patients were scheduled for TAPP repair using double separate meshes with tacker fixation. Group B: Twenty patients were scheduled for TAPP repair using a single large butterfly mesh without fixation. All patients subjected to pre-operative assessment in the form of full history, examination, laboratory investigations, cardio-pulmonary assessment and abdominal ultrasonography and all patients provided a written consent to participate in the study. The study was approved by the Ethics Board of Al-Azhar University.

\section{Surgical Technique}

Under general anesthesia, patients were placed in supine position, with a $10^{\circ}$ Trendelenburg tilt with both arms in adduction position to patient sides. The surgeon stands on the contra-lateral side of the hernia, and the assistant stands opposite to the surgeon and equipments were placed to the foot of the table (Fig. 1). After pneumoperitoneum, using veress needle three trocars were inserted: first, one $10 \mathrm{~mm}$ supra umbilical for $30^{\circ}$ scope and 2nd and 3rd were 5-10 
$\mathrm{mm}$ for the instruments and mesh on both sides of the optic trocar.

Laparoscopic exploration was done to identify the anatomical landmarks, the site and type of hernia, triangle of pain and triangle of doom to avoid them during mesh fixation.

In group A, we began preperitoneal dissection by opening the peritoneum by two separate incisions on both sides using the scissor and diathermy, starting 2 $\mathrm{cm}$ just above the anterior superior iliac spine until the medial umbilical ligament on the same side (Fig. 2 ). Then we continued the dissection to create lateral and medial preperitoneal pockets lateral and medial to the spermatic cord avoiding injury of inferior epigastric vessels (Fig. 3).
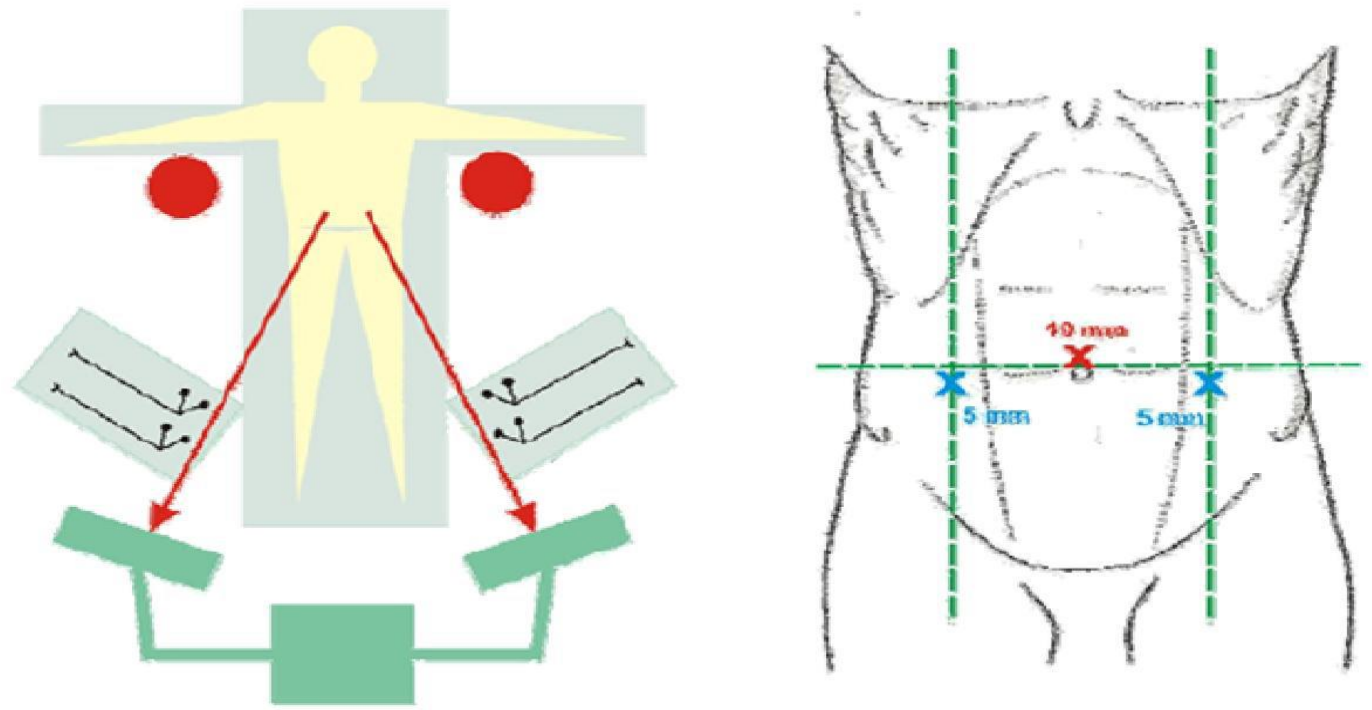

Fig (1): Operating room setup and trocar placement.

Medial dissection is carried out until the exposure of Cooper's ligament and the pubis. Then, dissection of the hernia sac was done taking care to protect the vas deferens and spermatic vessels from injury (Fig. 4).

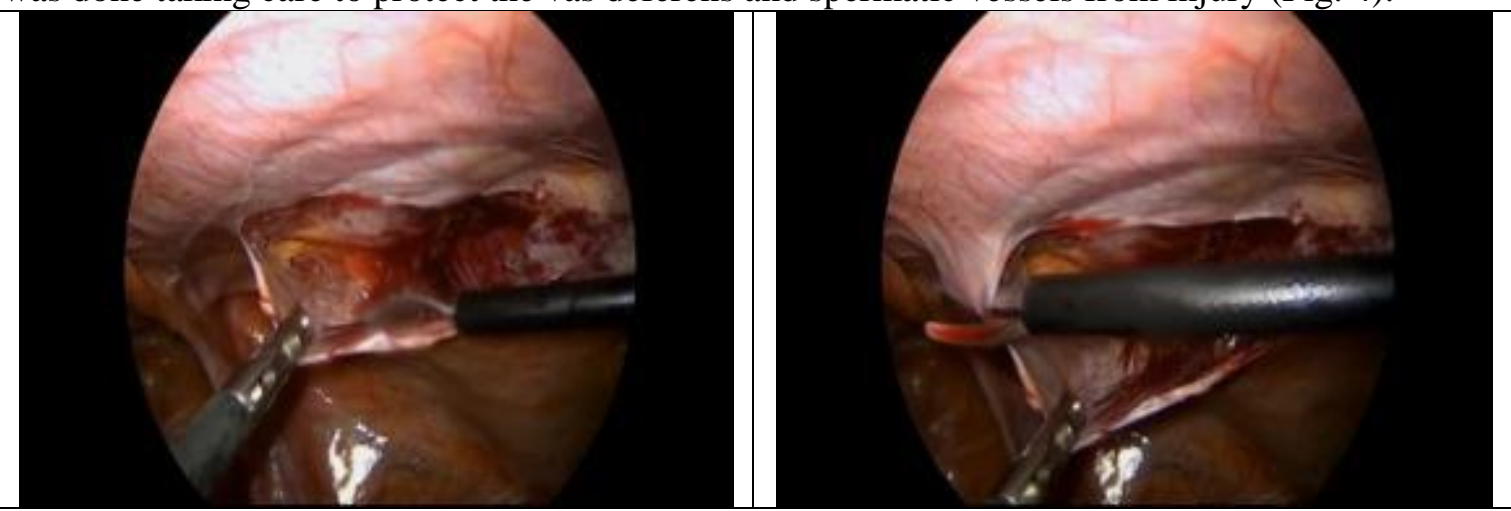

Fig (2): Peritoneal incision starting $2 \mathrm{~cm}$ above ASIS to the medial umbilical ligament.

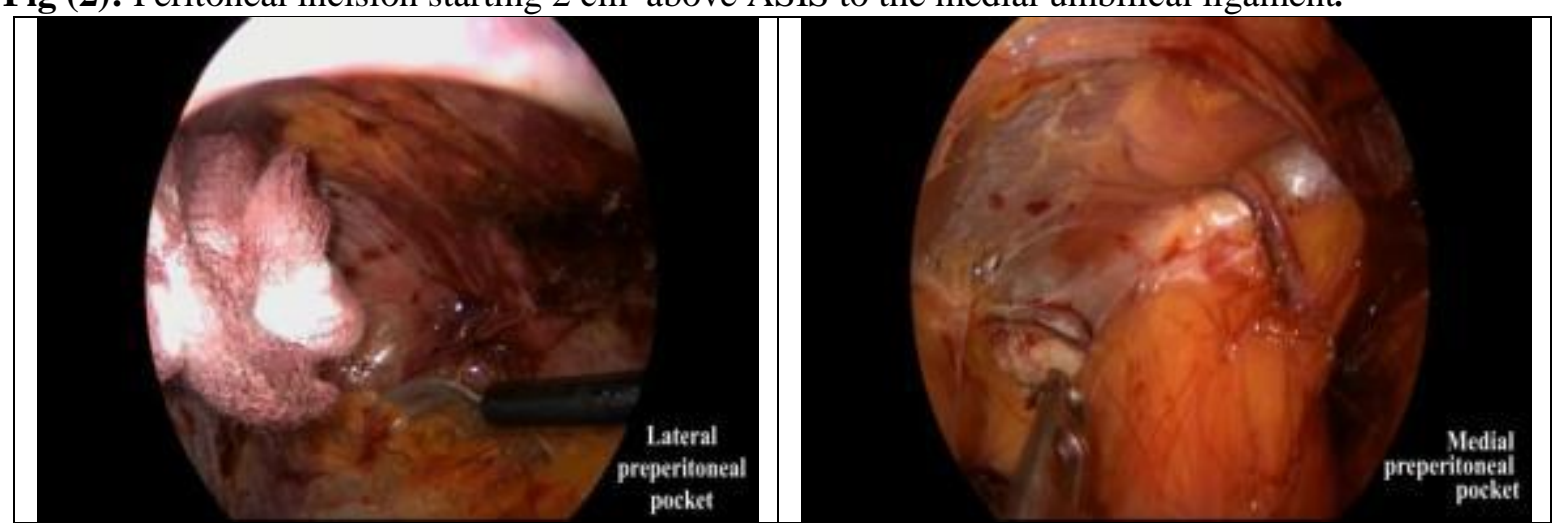

Fig (3): Creating lateral and medial preperitoneal pockets. 

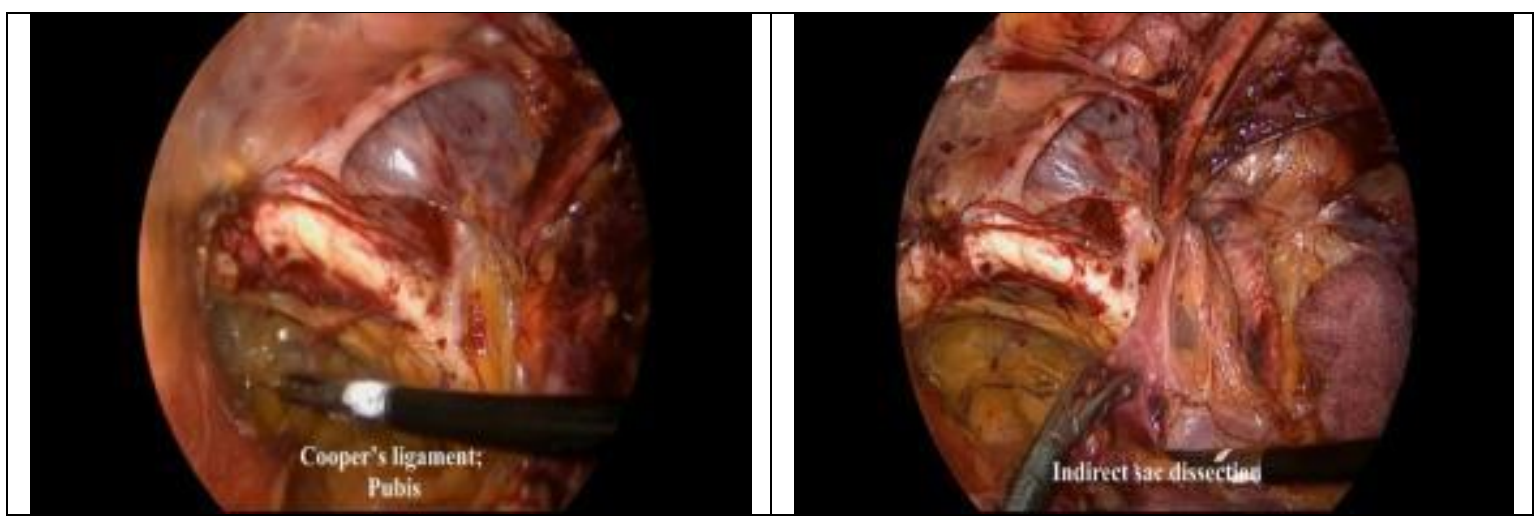

Fig (4): Exposure of Cooper's ligament and dissection of the hernia sac.

After complete dissection and identification of pain triangle and vascular triangle (Fig. 5), two separate polypropylene meshes $12 \times 15 \mathrm{~cm}$ were rolled and inserted through the $10 \mathrm{~mm}$ port, then placed in the dissected space to cover the hernia defect and fixed by using stapler. The first tack was fixed at the level of Cooper's ligament and then $2 \mathrm{~cm}$ above the level of anterior superior iliac spine then on both sides of the inferior epigastric vessels. Closure of the peritoneum was done by using a $2 / 0$ absorbable monofilament suture.

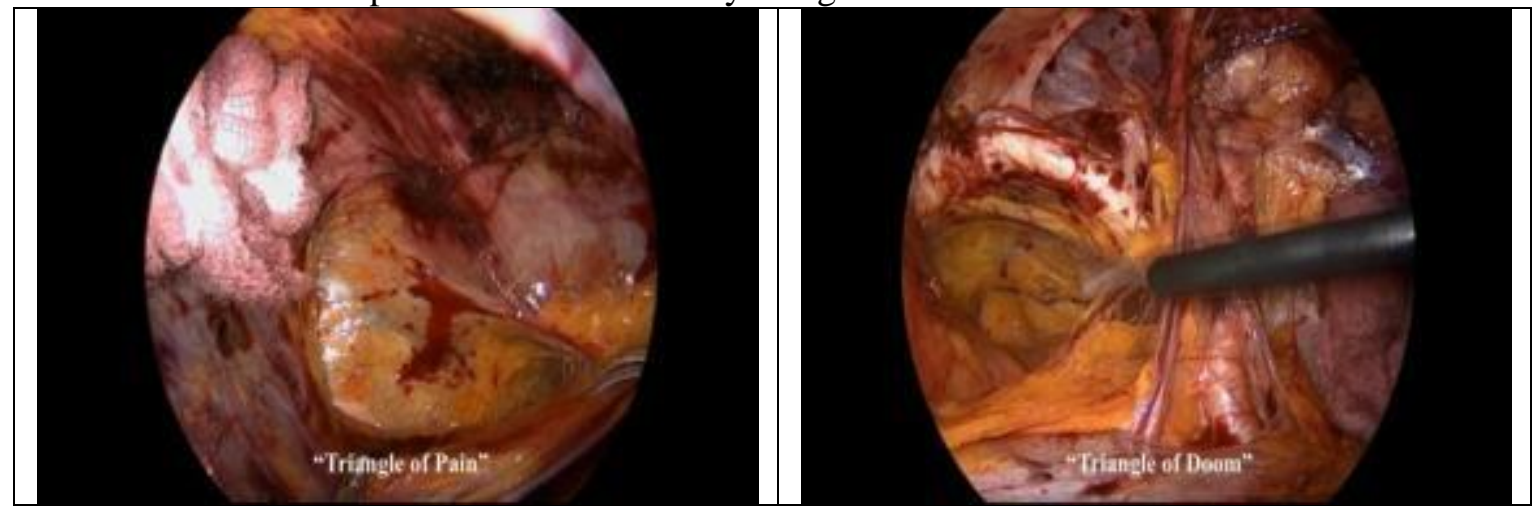

Fig (5): Identification of pain triangle and vascular triangle.

In group B, a similar technique of dissection was done. Then the space of Bogros was opened anterior to the bladder by extended medial dissection on both sides and both inguinal fossae were joined together (Fig. 6). The anatomic landmarks were identified taking care of urinary bladder, epigastric vessels, external iliac vessels, corona mortis vein, spermatic vessels and vas deferens.

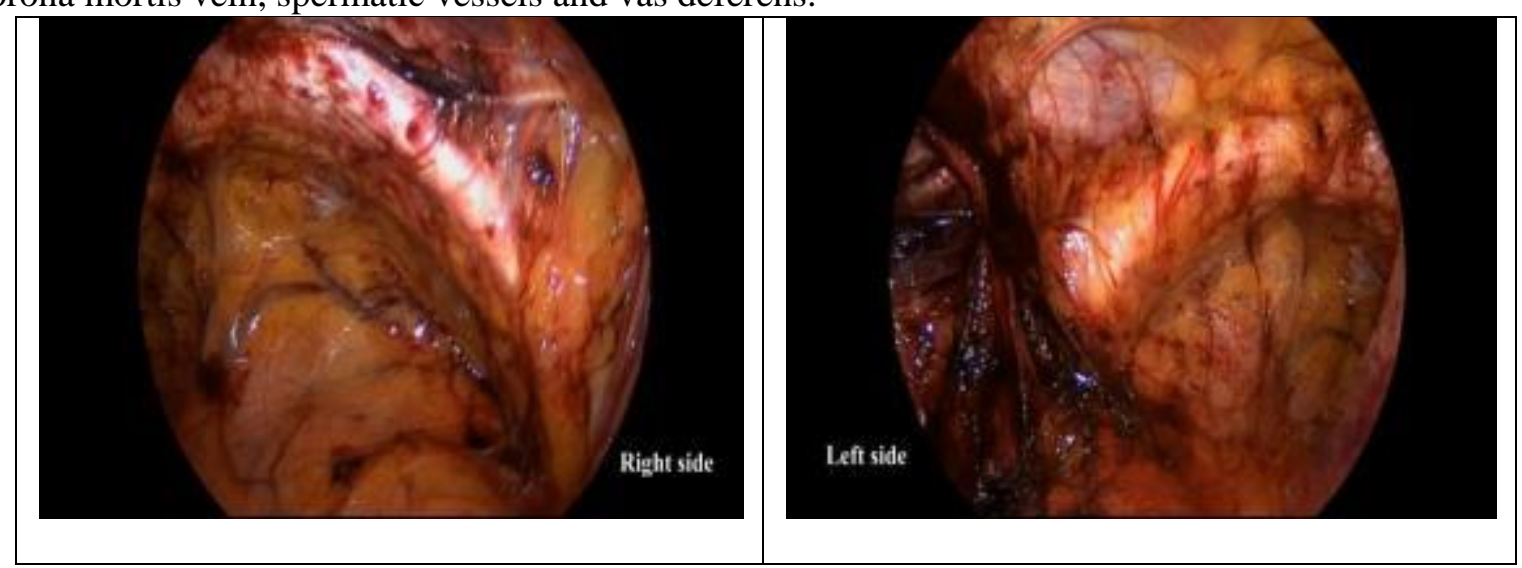

Fig (6): Dissection of the space of Bogros.

A large $30 \times 12 \mathrm{~cm}$ Polypropylene mesh was trimmed and designed to be butterfly in shape. Each wing measured $12 \times 13 \mathrm{~cm}$ and the isthmus was about $4 \times 6 \mathrm{~cm}$. The mesh is rolled in cigar shape manner and fixed by two stay sutures then introduced through $10 \mathrm{~mm}$ port to one space and pulled from the opposite side to cover both spaces (Fig. 7). The mesh is unrolled by removing the stay sutures and appropriately expanded to cover the dissected space without fixation (Fig. 8). Peritoneal closure was done by using a $2 / 0$ absorbable monofilament suture. 


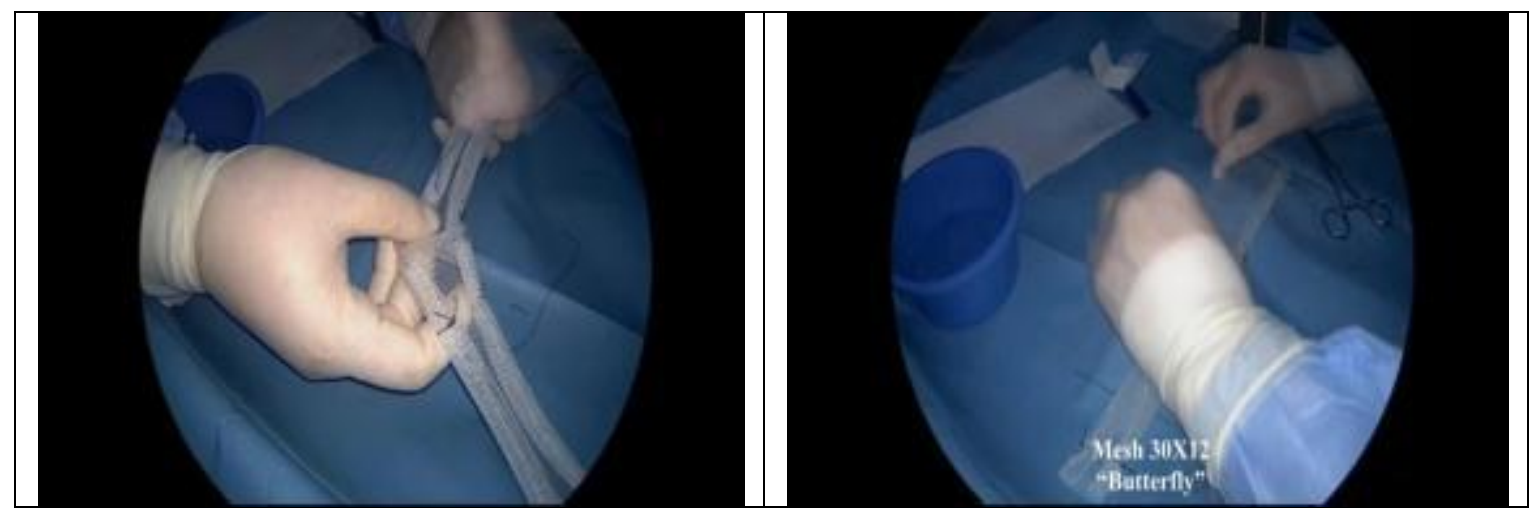

Fig (7): Polypropylene mesh is trimmed and designed to be butterfly in shape.

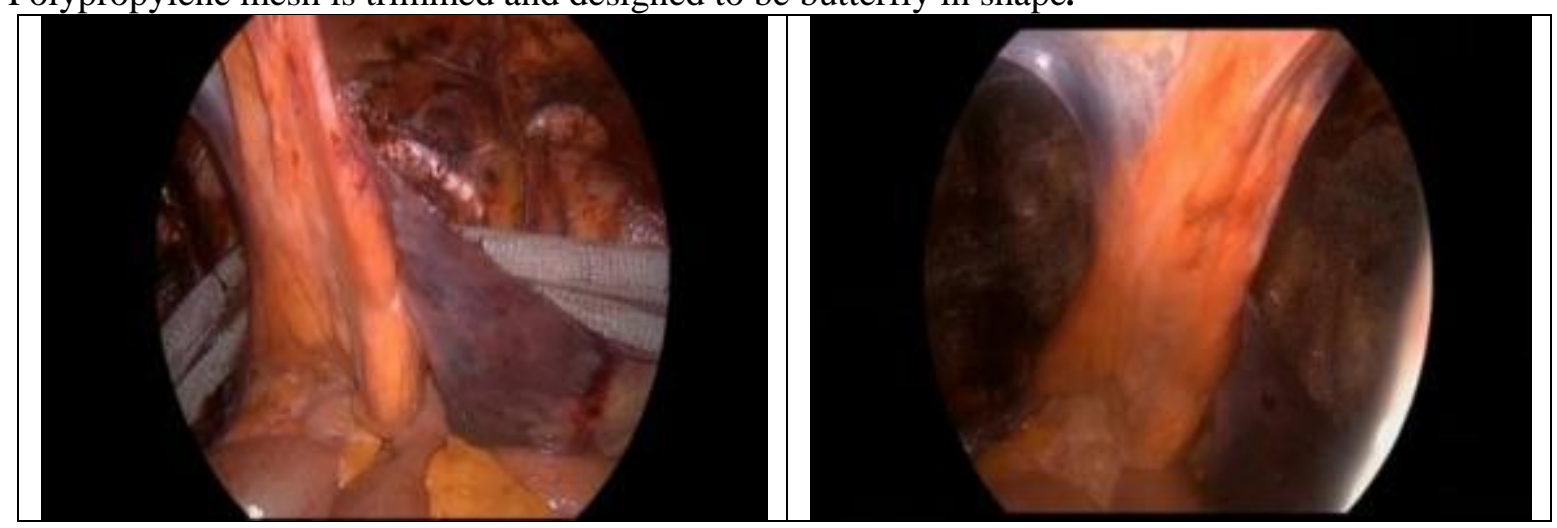

Fig (8): Mesh is expanded to cover the dissected space without fixation.

Post-operatively, patients were discharged to home after 24 - 48 hours and followed up after one week, one month, 6 months, 12 months, and 18 months. During each visit symptoms and signs of postoperative complications were detected and recorded. The preoperative data collected for each group included age, sex, BMI, hernia type and operative time. Post-operative data included hospital stay, post-operative pain score, postoperative wound complications and recurrence rate, and then we compared the data of both groups.

\section{Statistical Analysis}

Results in both groups were compared and statistically analyzed using two by two table, Chisquare was used when appropriate. $\mathrm{P}$ value $\leq 0.05$ is considered significant.

\section{RESULTS}

This study included 40 patients diagnosed as bilateral inguinal hernia (32 males and 8 females), the mean age of group A was $38.68 \pm 17.56$ and for group $\mathrm{B}$, it was $40.13 \pm 15.37$ years. The mean BMI was $31.27 \pm 43.61$ for group A and $30.74 \pm$ 61.72 for group B with no significant statistical difference between the two groups. The mean operative time (minutes) for Group A was $72.56 \pm$
8.42 minutes, while it was $64.37 \pm 12.52$ minutes for group B with a significant statistical difference. The mean hospital stay for group A was $1.8 \pm 0.52$ and it was $1.7 \pm 0.83$ days for group B with no significant statistical difference (Table 1).

All cases in both groups were done laparoscopically without conversion to open. All patients in this study had a follow up for at least 1 year after surgery. In the present study, intraoperative bleeding happened in one patient (5\%) in group A without the need for blood transfusion, while two patients (10\%) in group B had bleeding also without the need for blood transfusion. The cause of bleeding in group A was injury of inferior epigastric artery in one patient $(5 \%)$. In group B, the source of bleeding was the peri-vesical fat. Scrotal hematoma reported in two cases $(10 \%)$ in group A and in one case $(5 \%)$ in group B. There was no post-operative port site infection, bladder or bowel injury or wound hematoma. The median pain score at the 1 st 6 hours was 3.62 for group A and 2.17 for group B with a significant statistical difference. There was one recurrence in group A on the left side after 8 months and no recurrences were demonstrated in group B (Table 2). 
Table (1): showing Preoperative data in the study groups (sex, age and BMI), operative time and hospital stay

\begin{tabular}{|c|c|c|c|}
\hline & Group A (two meshes) & Group B (single mesh) & $\boldsymbol{p}$ - value \\
\hline Age & $40.13 \pm 15.37$ & $38.68 \pm 17.56$ & NS \\
\hline Sex M/F & $15 / 5$ & $17 / 3$ & NS \\
\hline BMI & $31.27 \pm 43.61$ & $30.74 \pm 61.72$ & NS \\
\hline Operative time (minutes) & $72.56 \pm 8.42$ & $64.37 \pm 12.52$ & $<0.01$ \\
\hline Hospital stay (days) & $1.7 \pm 0.83$ & $1.8 \pm 0.52$ & NS \\
\hline
\end{tabular}

Table (2): showing the operative and post-operative complication for both groups.

\begin{tabular}{|c|c|c|c|}
\hline & Group A (two meshes) & Group B ( (single mesh & $p$-value \\
\hline $\begin{array}{c}\text { Intraoperative } \\
\text { Bleeding }\end{array}$ & $1(5 \%)$ & $2(10 \%)$ & NS \\
\hline Scrotal hematoma & $2(10 \%)$ & $1(5 \%)$ & NS \\
\hline Port site infection & $0(0 \%)$ & $0(0 \%)$ & NS \\
\hline Bladder injury & $0(0 \%)$ & $0(0 \%)$ & NS \\
\hline Wound hematoma & $0(0 \%)$ & $0(0 \%)$ & NS \\
\hline Pelvic hematoma & $0(0 \%)$ & $0(0 \%)$ & NS \\
\hline Pain score (6 h) & 3.62 & 2.17 & $<0.05$ \\
\hline $\begin{array}{c}\text { Recurrence within } \\
\text { the 1st year }\end{array}$ & $1(5 \%)$ & $0(0 \%)$ & NS \\
\hline
\end{tabular}

There was a significant statistical difference regarding the cost between the 2 groups as the cost of a single large mesh was $35 \%$ to $42 \%$ lower than that of 2 separate meshes.

\section{DISCUSSION}

Even after many studies done in recent years, no consensus has been achieved on the surgical technique of inguinal hernia repair ${ }^{(\mathbf{1 3})}$.

Compared with open repairs, laparoscopic inguinal hernia repair has some advantages, such as, lower score of post-operative pain, a shorter recovery time, earlier return back to the daily activities and work, better cosmetic results and lower recurrence rate. Some authors reported a high morbidity rate after bilateral hernia repair in comparison with unilateral hernia repair. Others reported that the morbidity rate for bilateral hernia repair when a tension free repair is performed was lower than unilateral hernia repair for each side. From the morbidity point of view, endoscopic technique for bilateral hernia repair is more effective with less morbidity rate than conventional repair, less post-operative pain, rapid recovery, lower recurrence rate ${ }^{(14)}$.

When the laparoscopic technique is used to repair a clinically diagnosed unilateral inguinal hernia, it is possible to also explore the contralateral side. In 10-25\% of cases, an asymptomatic, pre-operatively an apparent, occult inguinal hernia is identified on the other side. Accordingly, contralateral occult inguinal hernia found at the time of laparoscopic transabdominal preperitoneal patchplasty (TAPP) repair should also be repaired (15). In laparoscopic inguinal hernial repair, both hernial orifices can be observed clearly and tension free mesh repair carried out effectively ${ }^{(4)}$.
In bilateral inguinal hernia cases, laparoscopic surgery has many advantages as it can be done through the same incisions as in unilateral laparoscopic inguinal hernia repair without the need for additional incision ${ }^{(4)}$.

In the present study, 40 patients diagnosed as bilateral inguinal hernia (32 males and 8 females), were divided into 2 groups A \& B, where TAPP hernia repair was done with single large mesh in group A and with 2 separate meshes for group B. The mean age of group A \& B were $38.68 \pm 17.56$ and $40.13 \pm 15.37$ respectively. The mean BMI was $31.27 \pm 43.61$ and $30.74 \pm 61.72$ for group A \& B respectively.

In this study, we reported $64.37 \pm 12.52$ minutes as mean operative time for bilateral hernia repair with single large mesh (group A), and 72.56 \pm 8.42 minutes for 2 separate meshes group (group B), with a significant statistical difference. In a study done by Jacob et al. ${ }^{(16)}$, the main operative time for bilateral laparoscopic hernia repair (TAPP) with 2separate meshes was 73.99 minutes which was similar to the operative time for group B in this study. Feliu et al. ${ }^{\left({ }^{(9)}\right.}$ reported $48.8 \pm 10.8$ minutes operative time for laparoscopic bilateral repair with 2 meshes which is shorter than our results for group B. Agresta et al. ${ }^{(17)}$ reported operative time of $43.5 \pm 13.2$ minutes for 2 mesh group, this results near the results of Feliu et al. ${ }^{(9)}$ and shorter than our results. In a study done by Kalpesh et al. ${ }^{(15)}$, While bilateral TAPP surgery with 2 meshes was performed, 23 cases (76.67\%) were finished within 2 hours and 6 cases (20\%) 
surgery took 3 hours to be finished. These results was longer than our results. Chalkoo et al. ${ }^{(18)}$ reported mean operative time of 48.5 minutes for bilateral TAPP repair with 2 meshes, which was shorter than our results.

The mean hospital stay in the present study was $1.8 \pm 0.52$ days for group $\mathrm{A}$, and $1.7 \pm 0.83$ days for group $B$, with no significant statistical difference. Jacob et al. ${ }^{\left({ }^{(16)}\right.}$ reported 2.08 days as a mean hospital stay for bilateral TAPP surgery with 2 meshes, while Kalpesh et al. ${ }^{(15)}$ reported hospital stay as, 22 patients $(73.33 \%)$ were discharged within 36 hours of surgery and all 30 (100\%) patients were discharged within 3 days of surgery. Hospital stay was 2 days in a study done by Zdravko et al. ${ }^{(19)}$ for bilateral hernia repair with 2 meshes.

In the present study, bleeding was reported in 1 patient (5\%) in group A due to injury of inferior epigastric artery, and in $2(10 \%)$ patients in group B the source of bleeding was the peri-vesical fat, without the need for blood transfusion. There was no post-operative port site infection, bladder or bowel injury or wound hematoma

Zdravko et al. ${ }^{(19)}$ reported post-operative complication as seroma occurred in 6 patients (4.62\%), one of them needed aspiration while the other 5 patients treated conservatively. Wound infection happened in 2 patients $(1.54 \%)$ and recurrence was reported in 1 patient $(0.77 \%)$. No recorded cases of neuralgia or scrotal hematoma. Jacob et al. (16) reported his post-operative complications as, intra-operative bleeding was 52 $(1.21 \%)$, urinary bladder injury was $4(0.09 \%)$, seroma at $155(3.61 \%)$, wound infection at $3(0.07$ $\%)$, intestinal injury at $4(0.04 \%)$ and reoperation was done at $84(1.96 \%)$. While the study of Feliu et al. ${ }^{(9)}$ reported, hematoma in 3 patients (4\%), seroma in 3 patients (4\%), bleeding in 1 patient $(1.3 \%)$, no reported cases of wound infection or urinary bladder or intestinal injuries

Pain score in this study, at the 1st 6 hours was higher in group B than group A, and reported as 2.17 and 3.62 for group A and B respectively with a significant statistical difference. The present study reported that the cost of a single large mesh was $35 \%$ to $42 \%$ lower than that of 2 separate meshes, with a significant statistical difference.

\section{REFERENCES}

1.LeBlanc K (2003): Hernias: Inguinal and Incisional. Lancet, 362: 1561-1571.

2. Thumbe VK, Evans DS (2001): To repair or not to repair incidental defects found on laparoscopic repair of groin hernia. Surg Endosc., 15: 47-49.

3. Griffin KJ, Harris S, Tang TY et al. (2010): Incidence of contralateral occult inguinal hernia found at the time of laparoscopic trans-abdominal pre-peritoneal (TAPP) repair. Hernia, 14: 345-349.

4. Wauschkuhn CA, Schwarz J, Boekeler U et al. (2010): Laparoscopic inguinal hernia repair: gold standard in bilateral hernia repair? Results of more than 2800 patients in comparison to literature. Surg Endosc., 24: 3026-3030.

5.Simons MP, Aufenacker T, Bay-Nielsen M et al. (2009): European Hernia Society guidelines on the treatment of inguinal hernia in adult patients. Hernia, 13: 343-403.

6. Bittner R, Arregui ME, Bisgaard $\mathrm{T}$ et al. (2011): International Endohernia Society (IEHS)]. Guidelines for laparoscopic (TAPP) and endoscopic (TEP) treatment of inguinal hernia. Surg Endosc., 25: 2773-2843.

7. Gould J (2008): Laparoscopic versus open inguinal hernia repair. Surg Clin North Am., 88: 1073-1081.

8. Weber-Sánchez A, Weber-Alvarez P, GarteizMartínez D (2016): Laparoscopy and Bilateral Inguinal Hernias, J Surg Transplant Sci., 4 (1): 1019-1022.

9. Feliu X, Jaurrieta E, Viñas X et al. (2004): Recurrent inguinal hernia: a 10-year review. J J Laparoendosc AdvSurg Tech., 14: 362-367.

10.Schwab R, Schumacher O, Junge K (2008): Biomechanical analyses of mesh fixation in TAPP and TEP hernia repair. Surg Endosc., 22: 731-738.

11. Beattie GC, Rumar S, Nixon SJ (2000): Laparoscopic total extraperitoneal hernia repair: mesh fixation is unnecessary. J Laparoendosc Adv Surg., 10: 71-73.

12.Poobalan AS, Bruce J, Cairns W et al. (2003): A review of chronic pain after inguinal herniorrhaphy. Clin J Pain, 19: 48-54.

13.Takata MC, Duh QY (2008): Laparoscopic Inguinal Hernia Repair. Surgical Clinics of North America, 88: 157- 178.

14. Griffin KJ, Harris S, Tang TY et al. (2010): Incidence of contralateral occult inguinal hernia found at the time of laparoscopic trans-abdominal pre-peritoneal (TAPP) repair. Hernia, 14: 345-349.

15. Kalpesh HP, Jayesh BGl, Bhumika JP (2017): Managing bilateral inguinal hernia laparoscopically is it gold standard? Int Surg J., 4 (1): 296-299.

16.Jacob DA, Hackl JA, Bittner $R$ et al. (2015): Perioperative outcome of unilateral versus bilateral inguinal hernia repairs in TAPP technique: analysis of 15,176 cases from the Herniamed Registry, Surg Endosc., 29: 3733-3740.

17.Agresta F, Alice M, Luca A et al. (2016): Laparoscopic Tapp Inguinal Hernia Repair. Mesh Fixation with Absorbable Tacks Initial Experience. J Minim Invasive Surg Sci., 16: 35-38.

18. Chalkoo M, Mujahid A, Hilal M (2016): Laparoscopic Trans-abdominal Preperitoneal Mesh Hernioplasty: A Medical College Experience. Surgical Science, 7: 107113.

19. Zdravko $P$, Mislav $R$, Zenon $P$ et al. (2011): Laparoscopic Transabdominal Preperitoneal Approach for Inguinal Hernia Repair: A FiveYear Experience at a Single Center. Surg Today, 41: 216-221. 